\title{
openheart Sex disparities in the presentation, management and outcomes of patients with acute coronary syndrome: insights from the ACS QUIK trial
}

\author{
Haitham Khraishah (D) , ${ }^{1}$ Barrak Alahmad, ${ }^{2}$ Abdulhamied Alfaddagh, ${ }^{3}$ \\ Sun Young Jeong, ${ }^{1}$ Njambi Mathenge, ${ }^{4}$ Mohamad Bassam Kassab, ${ }^{4}$ Dhaval Kolte, ${ }^{4}$ \\ Erin D Michos (D) , ${ }^{3}$ Mazen Albaghdadi ${ }^{4,5}$
}

To cite: Khraishah $\mathrm{H}$, Alahmad B, Alfaddagh A, et al. Sex disparities in the presentation, management and outcomes of patients with acute coronary syndrome: insights from the ACS QUIK trial. Open Heart 2021;8:e001470.

doi:10.1136/

openhrt-2020-001470

Received 2 0ctober 2020 Revised 22 December 2020 Accepted 28 December 2020

Check for updates

(c) Author(s) (or their employer(s)) 2021. Re-use permitted under CC BY-NC. No commercial re-use. See rights and permissions. Published by BMJ.

1 Department of Medicine, Beth Israel Deaconess Medical Center, Harvard Medical School, Boston, Massachusetts, USA

${ }^{2}$ Environmental Health Department, T H Chan School of Public Health, Harvard University, Boston, Massachusetts, USA

${ }^{3}$ Division of Cardiology, Johns Hopkins University School of Medicine, Baltimore, Maryland, USA

${ }^{4}$ Division of Cardiology, Massachusetts General Hospital Boston, Massachusetts, USA ${ }^{5}$ Division of Cardiology, Peter Munk Cardiac Centre, Toronto General Hospital, University of Toronto, Toronto, Ontario, Canada

Correspondence to Dr Haitham Khraishah; hkhraish@bidmc.harvard.edu

\section{ABSTRACT}

Aims Our aim was to explore sex differences and inequalities in terms of medical management and cardiovascular disease (CVD) outcomes in a low/middleincome country (LMIC), where reports are scarce. Methods We examined sex differences in presentation, management and clinical outcomes in 21374 patients presenting with acute coronary syndrome (ACS) in Kerala, India enrolled in the Acute Coronary Syndrome Quality Improvement in Kerala trial. The main outcomes were the rates of in-hospital and 30-day major adverse cardiovascular events (MACEs) defined as composite of death, reinfarction, stroke and major bleeding. We fitted log Poisson multivariate random effects models to obtain the relative risks comparing women with men, and adjusted for clustering by centre and for age, CVD risk factors and cardiac presentation.

Results A total of 5191 (24.3\%) patients were women. Compared with men, women presenting with ACS were older ( $65 \pm 12$ vs $58 \pm 12$ years; $p<0.001$ ), more likely to have hypertension and diabetes. They also had longer symptom onset to hospital presentation time (median, 300 vs $238 \mathrm{~min} ; p<0.001$ ) and were less likely to receive primary percutaneous coronary intervention for STelevation myocardial infarction (45.9\% vs $49.8 \%$ of men, $\mathrm{p}<0.001)$. After adjustment, women were more likely to experience in-hospital (adjusted relative risk $(\mathrm{RR})=1.53$; $95 \% \mathrm{Cl} 1.32$ to $1.77 ; \mathrm{p}<0.001)$ and 30 -day MACE (adjusted RR=1.39; $95 \% \mathrm{Cl} 1.23$ to $1.57, \mathrm{p}<0.001$ ). Conclusion Women presenting with ACS in Kerala, India had greater burden of CVD risk factors, including hypertension and diabetes mellitus, longer delays in presentation, and were less likely to receive guidelinedirected management. Women also had worse in-hospital and 30-day outcomes. Further efforts are needed to understand and reduce cardiovascular care disparities between men and women in LMICs.

\section{INTRODUCTION}

Cardiovascular disease (CVD) is the leading cause of death in women worldwide, claiming an estimated 6 million lives each year. ${ }^{2}$ The burden of CVD has shifted globally toward

\section{Key questions}

What is already known about this subject?

- Reports from high-income countries demonstrate gender disparities in acute coronary syndrome (ACS) presentation and management with women being disadvantaged. However, conflicting data exist in regards to short-term and intermediate-term outcomes. Scarce reports are available on sex disparities in low/middle-income countries (LMICs).

What does this study add?

- Limited evidence from LMICs demonstrates gender disparities in the presentation and management for women with ACS. However, prior studies have reported conflicting data regarding gender disparities in the incidence of adverse clinical outcomes including major adverse cardiovascular events, inhospital mortality and long-term prognosis. In this large study of patients with ACS from Kerala, India, we now show that women had higher in-hospital and 30-day mortality even after adjusting for possible confounders, such as age, cardiac risk factors and hospital of presentation.

How might this impact on clinical practice?

- These results are important for clinicians, cardiologists and global health experts. Improved awareness, research, advocacy and guidelines related to women's heart health are needed, especially in LMICs, where the gap is larger.

low/middle-income countries (LMICs), which disproportionately carry $80 \%$ of the burden of GVD. The burden of CVD in LMICs is only expected to rise as these countries progress through the epidemiological transition. ${ }^{3}$ Studies predominantly from high-income countries (HICs) have shown that women with acute coronary syndromes (ACS) are less likely to receive guideline-recommended pharmacotherapy and undergo invasive angiography and 
percutaneous coronary intervention (PCI) compared with men..$^{5-8}$ These diagnostic and therapeutic disparities in cardiovascular care are associated with worse outcomes and represent opportunities for quality improvement initiatives to narrow the sex gap in ACS outcomes. ${ }^{9}$

India has one of the highest burden of atherosclerotic CVD in the world where women account for $40 \%$ of all CVD deaths. ${ }^{4} 1011$ Studies evaluating sex differences in CVD in India are limited but indicate an alarming steady increase in death rates in women and a consistent pattern of sex-associated differences in presentation and management. ${ }^{12-15}$ However, inconsistent data exist on whether those differences ultimately impact in-hospital and longterm outcomes among Indian women with ACS. ${ }^{1} 1314$ Using data from the Acute Coronary Syndrome Quality Improvement in Kerala (ACS QUIK) randomised clinical trial, we aimed to examine the impact of sex on the clinical presentation, management and outcomes in a contemporary, large population of patients with ACS in an LMIC.

\section{METHODS}

\section{Study population}

The ACS QUIK trial was a large pragmatic, clusterrandomised, stepped-wedge clinical trial examining the effect of a quality improvement toolkit intervention on major adverse cardiovascular events (MACEs) in patients with ACS. The design and primary results of the trial were published elsewhere. ${ }^{16}{ }^{17}$ Briefly, 63 hospitals in Kerala, India participated in a cluster-randomised, stepped-wedge clinical trial to evaluate the impact of a locally adapted quality improvement toolkit to improve ACS outcomes. ${ }^{18}$ The trial included 21374 patients with acute myocardial infarction (AMI) (ST-elevation myocardial infarction (STEMI) and non-ST-elevation myocardial infarction (NSTEMI)) enrolled between 10 November 2014 and 9 November 2016. Patients with unstable angina were excluded. In this analysis, we examined differences between women versus men presenting with AMI, in terms of baseline characteristics, management and relevant clinical outcomes.

The current analysis was approved by the Partners Healthcare Institutional Review Board, Boston, Massachusetts, USA and Biologic Specimen and Data Repository Information Coordinating Center (BioLINCC; National Heart, Lung and Blood Institute, Bethesda, Maryland, USA). HK and BA had full access to data in the study and take responsibility for its integrity and the data analysis.

\section{Outcomes}

The main outcome of ACS QUIK was 30-day MACE, defined as a composite of death, reinfarction, stroke and major bleeding. For this analysis, the main outcomes were the rates of in-hospital and 30-day mortality and MACE (as defined above). Major bleeding was defined according to the Global Utilization of Streptokinase and
Tissue Plasminogen Activator for Occluded Coronary Arteries criteria. ${ }^{19}$ Other outcomes assessed included in-hospital-incident heart failure and cardiac arrest.

\section{Covariates}

The choice of covariate adjustment in the models was based on parsimony and a priori clinical hypotheses to control for confounding. In all our models we adjusted for age (linearly), smoking or tobacco use (yes/no), hypertension and diabetes on presentation (yes/no), cardiac presentation status (STEMI)/NSTEMI), and whether PCI was done (yes/no). We also evaluated potential confounding by differences in cardiac care in the 63 hospitals and centres in a fixed effects model.

\section{Data analysis}

Continuous variables are summarised as mean $\pm \mathrm{SD}$ if normally distributed, and as median and IQR if not normally distributed. We used Student's t-test and MannWhitney U test to compare groups accordingly. Categorical variables are reported as numbers and percentages and were compared between groups using $\mathrm{X}^{2}$ or Fisher's exact test as appropriate.

Multivariable log Poisson regression models were used to assess the association between sex and in-hospital or 30-day outcomes and adjust for possible confounders. Associations were summarised using adjusted and unadjusted relative risks (RRs) and 95\% CIs. We reported both unadjusted (crude) and adjusted RRs for all outcomes. For the adjusted RRs, we applied generalised linear mixed-effects models with multivariable normal random effects, using penalised quasi-likelihood. This function enabled us to fit models with different family distributions and link functions (log Poisson) that are non-parametric, as compared with the linear mixedeffects that offer parametric estimates. We accounted for within-hospital clustering by fitting random intercepts for different hospitals. We reported the fixed coefficients which are interpreted as the overall RR comparing women with men. In a sensitivity analysis, we also report fixed effects (with dummy variables) to understand the effect of measured and unmeasured confounding by hospitals.

We examined effect measure modification by a number of variables. This was made possible by dichotomising continuous variables and looking at the RRs of in-hospital MACE comparing women with men within each category. The interaction was tested using the $\mathrm{p}$ value from the Wald test that corresponds to the interaction term coefficient in the full sample size.

All $\mathrm{p}$ values were two-sided with a significance level of $<0.05$. Data analyses were conducted using R V.3.5.2 ( $R$ Foundation for Statistical Computing, Vienna, Austria) and Stata/IC V.15.0 (StataCorp). Mixed-effects models were fitted using the 'nlme' $\mathrm{R}$ package and 'glmmpql' function. 
Table 1 Baseline characteristics of ACS QUIK patients stratified by sex

\begin{tabular}{|c|c|c|c|c|}
\hline & Total $(n=21374)$ & Women $(n=5191)$ & Men $(n=16183)$ & $P$ value \\
\hline Age, mean (SD) & $60(12)$ & $65(12)$ & $58(12)$ & $<0.001$ \\
\hline Transferred from another facility, no (\%) & $8401(39.3)$ & $1932(37.2)$ & $6469(40.0)$ & $<0.001$ \\
\hline No insurance, no (\%) & $15542(72.7)$ & $3814(73.5)$ & $11728(72.5)$ & 0.16 \\
\hline ST-elevation myocardial infarction, no (\%) & $13689(64.0)$ & $2846(54.8)$ & $10843(67.0)$ & $<0.001$ \\
\hline Symptom-to-door time, median (IQR), min & $246(118-830.5)$ & $300(127.5-990)$ & $238(115-780)$ & $<0.001$ \\
\hline Body weight, mean (SD), kg & $63.0(10.0)$ & $59.0(10.0)$ & $65(9)$ & $<0.001$ \\
\hline $\begin{array}{l}\text { Systolic blood pressure, mean (SD), mm } \\
\mathrm{Hg}\end{array}$ & $139(29)$ & $141(30)$ & $138(29)$ & $<0.001$ \\
\hline Heart rate, mean (SD), /min & $80(19)$ & $83(20)$ & $79(19)$ & $<0.001$ \\
\hline Initial troponin, median (IQR), ng/mL & $1.32(0.29-5.82)(n=9049)$ & $1.05(0.26-4.02)(n=2299)$ & $1.44(0.3-6.58)(n=6750)$ & $<0.001$ \\
\hline $\begin{array}{l}\text { Low-density lipoprotein cholesterol, mean } \\
\text { (SD), mg/dL }\end{array}$ & $123(41)(n=14830)$ & $127(43)(n=3578)$ & $121(40)(n=11252)$ & $<0.001$ \\
\hline Triglycerides, median (IQR), mg/dL & $121(90-165)(n=14860)$ & $122(91-161)(n=3578)$ & $121(89-166)(n=11282)$ & 0.98 \\
\hline Serum creatinine, median (IQR), mg/dL & $1.0(0.9-1.2)(n=13835)$ & $0.92(0.8-1.2)(n=3361)$ & $1.1(0.9-1.3)(n=10474)$ & $<0.001$ \\
\hline Fasting glucose, median (IQR), mg/dL & $127(102-176)(n=13398)$ & $134(106-188)(n=3286)$ & $125(100-172)(n=10112)$ & $<0.001$ \\
\hline Haemoglobin, mean (SD), mg/dL & $13(2)(n=20842)$ & $12.0(2.0)(n=5064)$ & $14.0(2.0)(15778)$ & $<0.001$ \\
\hline \multicolumn{5}{|l|}{ Killip class, n (\%) } \\
\hline I & $18459(86.4)$ & 4266 (82.2) & $14193(87.7)$ & $<0.001$ \\
\hline II-IV & $2914(13.6)$ & $925(17.8)$ & $1989(12.3)$ & \\
\hline \multicolumn{5}{|l|}{ Risk factors, n (\%) } \\
\hline Hypertension & $10042(47.0)$ & $3179(61.2)$ & $6863(42.4)$ & $<0.001$ \\
\hline Diabetes mellitus & $9484(44.4)$ & $2783(53.6)$ & $6701(41.4)$ & $<0.001$ \\
\hline History of tobacco use, no (\%) & $6614(30.9)$ & $168(3.2)$ & $6446(39.8)$ & $<0.001$ \\
\hline Peripheral arterial disease & $211(1.0)$ & $50(1.0)$ & $161(1.0)$ & 1.00 \\
\hline History of stroke & $470(2.2)$ & $137(2.6)$ & $333(2.1)$ & 0.014 \\
\hline \multicolumn{5}{|l|}{ Hospital type } \\
\hline Government $(n=9)$ & $7133(33.4 \%)$ & $1618(31.2 \%)$ & $5515(34.1 \%)$ & $<0.001$ \\
\hline Non-profit/charity $(n=12)$ & $5749(26.9 \%)$ & $1478(28.5 \%)$ & $4271(26.4 \%)$ & \\
\hline Private $(n=42)$ & $8492(39.7 \%)$ & $2095(40.4 \%)$ & $6397(39.5 \%)$ & \\
\hline \multicolumn{5}{|l|}{ Hospital size } \\
\hline Extra large $(>1000)(n=5)$ & $3560(16.7 \%)$ & $730(14.1 \%)$ & $2830(17.5 \%)$ & $<0.001$ \\
\hline Large $(501-1000)(n=15)$ & $8523(39.9 \%)$ & $2089(40.2 \%)$ & $6434(39.8 \%)$ & \\
\hline Medium $(201-500)(n=24)$ & $7415(34.7 \%)$ & $1779(34.3 \%)$ & $5636(34.8 \%)$ & \\
\hline Small $(\leq 200)(n=19)$ & $1876(8.8 \%)$ & $593(11.4 \%)$ & $1283(7.9 \%)$ & \\
\hline \multicolumn{5}{|l|}{ Presence of onsite cath lab } \\
\hline Installed during study $(\mathrm{n}=3)$ & $496(2.3 \%)$ & $136(2.6 \%)$ & $360(2.2 \%)$ & $<0.001$ \\
\hline № $(n=17)$ & $3552(16.6 \%)$ & $976(18.8 \%)$ & $2576(15.9 \%)$ & \\
\hline Yes $(n=43)$ & $17326(81.1 \%)$ & $4079(78.6 \%)$ & 13247 (81.9\%) & \\
\hline
\end{tabular}

ACS QUIK, acute coronary syndrome quality improvement in Kerala; IQR, interquartile range; SD, standard deviation.

\section{RESULTS}

\section{Baseline characteristics}

Of 21374 patients with ACS enrolled in the ACS QUIK trial, $5191(24.3 \%)$ were women. Table 1 shows the baseline characteristics of the study population by sex. Compared with men with ACS, women with ACS were older (mean \pm SD, $65 \pm 12$ vs $58 \pm 12$ years; $\mathrm{p}<0.001$ ), more likely to have hypertension $(61.2 \%$ vs $42.4 \%$; $\mathrm{p}<0.001)$, diabetes mellitus $(53.5 \%$ vs $41.4 \%$; $\mathrm{p}<0.001)$, and higher low-density lipoprotein cholesterol levels $(127 \pm 43$ vs $121 \pm 40 \mathrm{mg} / \mathrm{dL} ; \mathrm{p}<0.001)$. After symptom onset, women tended to present later to the hospital (median (IQR) time, 300 (12.7.5-990) vs $238(115-780) \mathrm{min} ; \mathrm{p}<0.001)$. Compared with men, women were less likely to present 
Table 2 In-hospital and on-discharge treatment patterns stratified by sex

\begin{tabular}{|c|c|c|c|c|}
\hline & \multicolumn{3}{|l|}{ No/total no (\%) } & \multirow[b]{2}{*}{$P$ value } \\
\hline & Total $(n=21374)$ & Women $(n=5191)$ & Men $(n=16$ 183) & \\
\hline \multicolumn{5}{|l|}{ Medications } \\
\hline In-hospital aspirin & 20 885/21 328 (97.9) & $5044 / 5171$ (97.5) & $15841 / 16157(98.0)$ & 0.033 \\
\hline In-hospital second antiplatelet & 20 973/21 347 (98.2) & $5071 / 5180(97.9)$ & 15 902/16 167 (98.4) & 0.028 \\
\hline In-hospital anticoagulant & 18 256/21 332 (85.6) & $4421 / 5177(85.4)$ & 13 835/16 $155(85.6)$ & 0.67 \\
\hline \multicolumn{5}{|l|}{ Studies and procedures } \\
\hline Echocardiography & $19725(92.3)$ & $4777 / 5191(92.0)$ & 14 948/16 $183(92.4)$ & 0.42 \\
\hline Diagnostic angiography & $12681(59.3)$ & 2638/5191 (50.8) & 10 043/16 $183(62.1)$ & $<0.001$ \\
\hline Thrombolysis (STEMI) & $3167 / 13689(23.1)$ & $635 / 2846(22.3)$ & 2532/10 843 (23.4) & 0.25 \\
\hline Door-to-needle time (STEMI), median (IQR), min & $\begin{array}{l}44(30-70) \\
(n=2888)\end{array}$ & $\begin{array}{l}45.5(30-75) \\
(n=594)\end{array}$ & $\begin{array}{l}43(29-70) \\
(n=2294)\end{array}$ & 0.001 \\
\hline Any reperfusion (STEMI) & $9872 / 13689(72.1)$ & $1942 / 2846(68.2)$ & $7930 / 10843(73.1)$ & $<0.001$ \\
\hline Rescue PCl & 1675/13 659 (12.3) & $308 / 2841$ (10.8) & 1367/10 818 (12.6) & 0.009 \\
\hline \multicolumn{5}{|l|}{ Discharge treatment and counselling } \\
\hline Discharge aspirin & 19 137/19 557 (97.9) & $4448 / 4603(96.6)$ & 14 689/14 954 (98.2) & $<0.001$ \\
\hline Discharge second antiplatelet agent & 19 201/19 591 (98.0) & $4509 / 4620(97.6)$ & 14 692/14 971 (98.1) & 0.026 \\
\hline
\end{tabular}

$\mathrm{ACE}$ inhibitor, angiotensin-converting enzyme inhibitor; ARB, angiotensin receptor blocker; IQR, interquartile range; $\mathrm{PCI}$, percutaneous coronary intervention; STEMI, ST-elevation myocardial infarction.

with STEMI $(67.0 \%$ vs $54.8 \%$; $<<0.001)$, but more likely to present with a Killip class II or greater $(17.8 \%$ vs $12.3 \%$; $\mathrm{p}<0.001)$. More women presented to small hospitals ( $<200$ beds) $(11.4 \%$ vs $7.9 \%$ of men; $\mathrm{p}<0.001)$, and women were less likely to present to extra-large hospitals (>1000 beds) ( $14.1 \%$ vs $17.5 \%$ of men, $\mathrm{p}<0.001)$.

\section{In-hospital and on-discharge management}

Among eligible individuals with no contraindications, the rates of in-hospital and discharge aspirin, adjuvant antiplatelet therapy (clopidogrel, prasugrel or ticagrelor), and statin prescription were high ( $>95 \%$ for all) and similar in men and women (table 2). The rate of in-hospital $\beta$-blockers, ACE inhibitors or angiotensin receptor blockers, or anticoagulant use did not differ by sex. Compared with men with ACS, women were less likely to undergo diagnostic coronary angiography $(50.8 \%$ vs $62.1 \%$ of men, $\mathrm{p}<0.001)$ or PCI ( $39.8 \%$ vs $51.4 \%$ in men, $\mathrm{p}<0.001)$ during hospitalisation. Women presenting with STEMI were less likely to receive primary PCI $(45.9 \%$ vs $49.8 \%$ of men, $\mathrm{p}<0.001)$ and had longer median door-to-balloon times $(90(60-270)$ vs $80(55-180) \mathrm{min}$ for men; $\mathrm{p}<0.001)$.

\section{Outcomes}

In the overall cohort, a total of 835 in-hospital MACE and 1247 30-day MACE events occurred among study participants (table 3). Compared with men, women were 53\% more likely to experience in-hospital MACE (adjusted RR=1.53; 95\% CI 1.32 to $1.77 ; \mathrm{p}<0.001$ ), and $67 \%$ more likely to die during their hospitalisation (adjusted $\mathrm{RR}=1.67 ; 95 \%$ CI 1.42 to 1.97 ; $\mathrm{p}<0.001$ ), even after taking into account age and potential confounders. Women were also more likely to experience in-hospital heart failure, stroke and cardiac arrest. Likewise, women had 39\% higher risk of 30-day MACE (adjusted $\mathrm{RR}=1.39 ; 95 \%$ CI 1.65 to $2.07, \mathrm{p}<0.001$ ), $48 \%$ higher risk of 30-day mortality (adjusted $\mathrm{RR}=1.48 ; 95 \%$ CI 1.29 to $1.70 ; \mathrm{p}<0.001)$ and a $50 \%$ higher risk of CVD mortality (adjusted RR=1.50; 95\% CI 1.30 to 1.72 ).

Subgroup analyses consistently showed worse MACE in women compared with men within the strata of age, 
Table 3 Risk of adverse clinical outcomes among patients with ACS for women compared with men

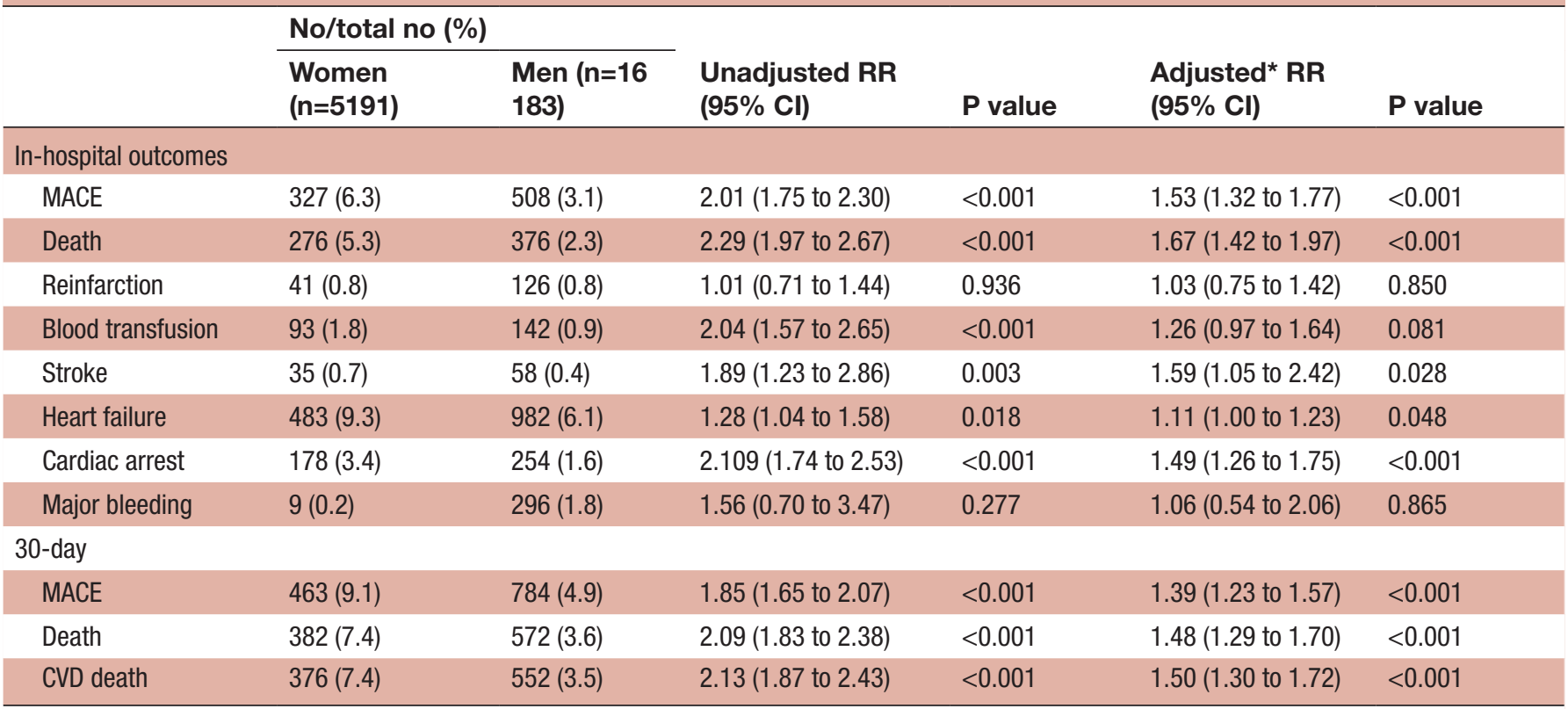

$\mathrm{RR}=$ relative risk from multivariate regression, multilevel analysis for women compared with men (reference). MACE included a composite of death, reinfarction, major bleeding and stroke.

*Models adjusted for age, smoking, diabetes, hypertension, MI subtype (STEMI vs NSTEMI) and PCI with random intercepts for centres. ACS, acute coronary syndrome; CVD, cardiovascular disease; MACE, major adverse cardiovascular event; MI, myocardial infarction; NSTEMI, non-ST-elevation myocardial infarction; PCI, percutaneous coronary intervention; STEMI, ST-elevation myocardial infarction.

diabetes, hypertension and smoking (figure 1). Compared with men, women also had worse in-hospital MACE whether or not they presented with STEMI or received PCI during their hospitalisation. The interaction terms in the full models between sex and these factors did not show statistical significance. In a sensitivity analysis using fixed effects model with dummy variables for 63 hospitals and centres, no appreciable difference was detected with the main model that had random intercepts, indicating a low risk of unmeasured confounding by different care providers (results not shown).

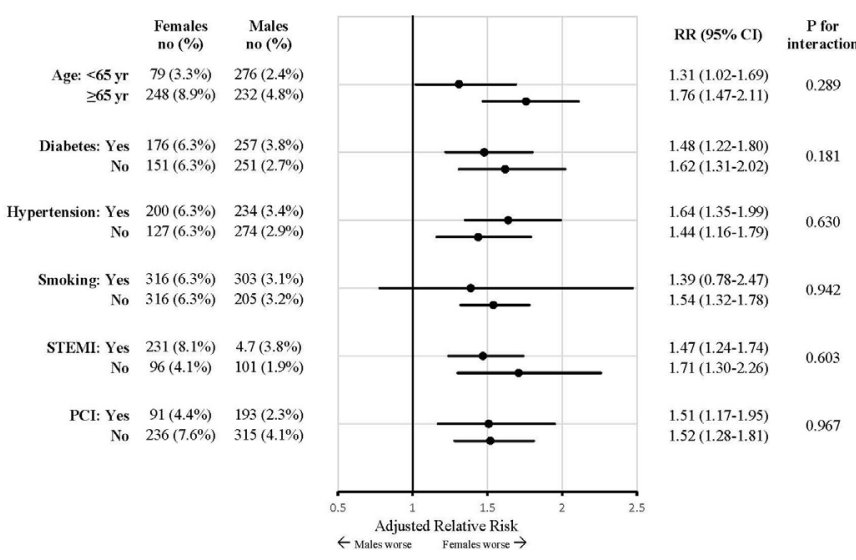

Figure 1 Adjusted relative risk (RR) of in-hospital major adverse cardiac events in selected subgroups comparing women with men (reference). $\mathrm{PCl}$, percutaneous coronary intervention; STEMI, ST-elevation myocardial infarction.

\section{DISCUSSION}

Our study examined sex differences in ACS outcomes in an LMIC (India) using data from the ACS QUIK trial, which included 21374 patients with ACS who presented to 63 hospitals in Kerala between 10 November 2014 and 9 November 2016. In this analysis, women presenting with ACS were older and more likely to have diabetes, hypertension and a history of stroke, but less likely to smoke compared with men. Women were also less likely to present with STEMI and more likely to present later after symptom onset and have heart failure on presentation. In-hospital and discharge medical management were similar for men and women; however, significant sex differences were evident in using potentially life-saving procedures such as primary PCI which was provided less often and with greater door-to-balloon time delays in women with STEMI compared with men. We also observed that women were more likely to experience in-hospital and 30-day MACE, even after adjustment for age, CVD risk factors, MI subtype, performance of PCI and other potential confounders. Additionally, significant sex disparities were evident after adjustment for these same covariates for in-hospital mortality, cardiac arrest and heart failure.

While there has been a steady increase in the research investigating sex differences in ACS presentation, management and outcomes in HICs, the evidence from LMICs is limited. Studies from HICs and LMICs demonstrate that women with ACS are older with a greater burden of comorbidities such as hypertension and diabetes mellitus, 
compared with men, which are consistent with the findings of the current study. ${ }^{5} 78$ 20-22 Furthermore, women with ACS tend to present later for medical attention after symptom onset and are less likely to receive optimal pharmacological and reperfusion therapy, even when eligible as seen in our study. ${ }^{6}$ However, prior studies have reported conflicting data regarding sex disparity in the incidence of adverse clinical outcomes including MACE, in-hospital mortality and long-term prognosis. ${ }^{6}{ }^{23}$ In this large study of patients with ACS from India, we now show that women have higher rates of in-hospital and 30-day MACE and mortality even after accounting for age and important comorbidities.

Limited resources in LMICs may worsen health disparities experienced by women and vulnerable populations. ${ }^{24}$ At a patient level, socioeconomic factors such as limited health literacy, medical insurance or lack of access to transportation may contribute to delays in the care of patients with ACS. ACS-related sex disparities have also been reported in studies from LMICs comparable with those from HICs. In a study of 1204 patients with ACS (253 women) in Egypt, the unadjusted in-hospital mortality was significantly higher in women compared with men (OR: $2.10 ; 95 \%$ CI 1.54 to 2.87). ${ }^{25}$ However, no significant difference in mortality was observed after adjusting for CVD risk factors. ${ }^{25}$ Another study from the Middle East-Gulf region looked at sex differences and STEMI outcomes. ${ }^{26}$ The study included a total of 15532 patients, 2033 of whom were women. The investigators found that women had higher in-hospital and 1-year mortality even after adjusting for common risk factors. ${ }^{26}$ The CRACE Study, which enrolled 1301 patients with ACS from 12 teaching hospitals across China, showed no significant differences in in-hospital mortality between men and women. ${ }^{27}$ Similar findings were reported in studies from Thailand and Malaysia. ${ }^{28} 29$

Despite the magnitude of atherosclerotic CVD in India, few studies have explored sex disparities in the management and outcomes of ACS in a large cohort of patients managed with contemporary ACS management strategies. The CREATE registry enrolled 20468 patients with ACS, of whom $23.6 \%$ were women, from 89 hospitals from 10 different regions in India. ${ }^{30}$ Similar to our study, women had lower rates of revascularisation and higher unadjusted all-cause mortality at 30 days. ${ }^{30}$ Unlike our results, mortality differences were attenuated after adjusting for treatment-related factors such as time-to-hospital presentation, revascularisation rates and utilisation of evidencebased medications. ${ }^{30}$ The DEMAT registry included 1565 patients with ACS (334 women) across 10 tertiary care centres in India between 2007 and 2008. ${ }^{13}$ Similar to our findings, in-hospital and on-discharge medical management was comparable among men and women, except for clopidogrel. In contrast to our study, no significant differences were noted in 30-day mortality or MACE (composite of death, rehospitalisation and cardiac arrest) after adjustment for potential confounders. ${ }^{13}$
Prior results from the Kerala-ACS ${ }^{14}$ registry, which included 125 centres (including the 63 centres involved in the ACS QUIK trial), did not find significant differences between men and women with ACS with respect to in-hospital mortality after adjustment for confounding factors. In the Kerala ACS registry, female patients with ACS were less likely to receive in-hospital aspirin $(92.4 \%$ vs $93.2 \%$, $\mathrm{p}=0.042)$ and $\beta$-blockers $(63.0 \%$ vs $66.6 \%, \mathrm{p}<0.001)$, but more likely to receive statins $(81.1 \%$ vs $78.1 \%, \mathrm{p}<0.001)$ compared with male patients. ${ }^{14}$ In addition, female and male patients were equally likely to receive in-hospital PCI, although the low frequency of PCI in both sexes $(12.4 \%$ in women vs $11.8 \%$ in men, $\mathrm{p}=0.202$ ). In comparison, the current study shows a higher proportion of male and female patients receiving aspirin, $\beta$-blockers and statins, with persistent sex differences in aspirin prescription. In ACS QUIK, the higher proportion of patients receiving PCI during their hospitalisation enabled for a sex-based comparison and showed a significantly lower likelihood of receiving PCI in women compared with men. These findings may provide potential mechanisms explaining the sex differences in in-hospital outcomes between the two studies. As the number of PCI-capable centres in India increased, the results of Kerala ACS and ACS QUIK highlight a sex-based care gap in coronary revascularisation that became more apparent in the periods $5-7$ years separating the two studies. The potential reasons for such gap need to be addressed in future research.

Both sex (biological differences) and gender (sociocultural differences) influence cardiovascular outcomes between women and men. Our findings can be partially explained by a higher risk factor profile in women along with late presentation and receiving less guidelinerecommended coronary reperfusion, compared with their male counterparts. However, this likely does not fully explain the mortality difference demonstrated in our study. Implicit gender bias towards female patients that precludes the administration of optimal care has been reported as a possible mechanism of gender disparities in cardiovascular care. ${ }^{31}$ Significant positive association between implicit bias and lower quality of care has been demonstrated in various studies. ${ }^{31}$ Maserejian $e t$ $a \hat{l}^{2}$ conducted a factorial experiment, using videotaped vignette of coronary artery disease with altering patients' sex, age and race. The group found that women, especially middle-aged, were diagnosed with the least confidence. ${ }^{32}$ Additionally, female patients were more likely to receive a diagnosis of mental health condition, compared with their male counterparts. ${ }^{32}$ In another US study, when compared with similar male patients, female patients with atherosclerotic CVD were also more likely to report that they had poor communication with their healthcare providers, that their doctors did not listen to them or respect them, and they had underutilisation of secondary prevention medications. ${ }^{33}$

The first step towards rectifying sex disparities in healthcare, in general, and CVD outcomes, in particular, is identifying and acknowledging the problem. This should 
be followed by raising awareness. 'Go Red For Women' and 'Women's Heart Alliance' are two campaigns aimed to improve awareness, advocacy and research related to women's heart health. They have been successful in drawing attention to women's heart health. We also need to implement delivery care models and specific guidelines to address women's cardiovascular health. ${ }^{34} 34$ Cardiovascular societies have already started to adopt guidelines for women, but this needs to be expanded. ${ }^{34}$ Similar efforts should be adopted internationally, especially in LMICs where the gap is even larger.

\section{Study strengths and limitations}

This study has a number of limitations. First, inferences from this study should be interpreted within the local context of the study population. It is likely that the results may not be generalisable to other populations where other factors could modify the nature of the relationship between sex and CVD outcomes. Second, we were unable to obtain information on socioeconomic resources (eg, income, education and others). We cannot rule out the possibility that these or other factors may contribute to the treatment and outcome disparities that we observed. Furthermore, control for smoking was not optimal as we did not have information on long-term use (eg, packyears) leaving a room for residual confounding. Finally, the interaction analyses were possibly underpowered to detect significant differences in effect estimates. On the other hand, our study had a number of important strengths including a large ACS sample from an LMIC with information on a number of other important confounding comorbidities and details regarding cardiac presentation and clinical management strategies that could be accounted for in our analysis.

\section{CONCLUSION}

Our study confirms a higher CVD risk profile, delayed presentation and suboptimal medical care in women presenting with ACS in Kerala, India. Women were also found to have higher in-hospital and 30-day MACE, even after adjustment for potential confounders. Women around the globe, and especially in LMIC, face sex-based societal and health inequities leading to sex differences in healthcare seeking behaviour and implicit care provider bias. Our study underscores the need to explore and mitigate these gaps through education, research, medical care and broader health policy. A better understanding of these differences is crucial in improving cardiovascular outcomes among women worldwide, especially in LMICs.

\section{Twitter Haitham Khraishah @HaithamKhraisha}

Contributors All authors contributed to the conception and design of the work. HK, $B A$ and MA then contributed to the acquisition, analysis or interpretation of data for the work. HK, BA, AA and MA drafted the manuscript. All listed authors had critically revised the manuscript. All gave final approval and agree to be accountable for all aspects of work ensuring integrity and accuracy.

Funding The authors have not declared a specific grant for this research from any funding agency in the public, commercial or not-for-profit sectors.
Competing interests None declared.

Patient consent for publication Not required.

Ethics approval The ACS QUIK trial received Institutional Review Board (IRB) approval from the ethics committees of the participating centerscentres and was approved by the Indian Health Ministry Screening Committee.

Provenance and peer review Not commissioned; externally peer reviewed.

Data availability statement Data are available in a public, open access repository. Data obtained from Biologic Specimen and Data Repository Information Coordinating Center (BioLINCC).

Open access This is an open access article distributed in accordance with the Creative Commons Attribution Non Commercial (CC BY-NC 4.0) license, which permits others to distribute, remix, adapt, build upon this work non-commercially, and license their derivative works on different terms, provided the original work is properly cited, appropriate credit is given, any changes made indicated, and the use is non-commercial. See: http://creativecommons.org/licenses/by-nc/4.0/.

ORCID iDs

Haitham Khraishah http://orcid.org/0000-0003-0003-5089

Erin D Michos http://orcid.org/0000-0002-5547-5084

\section{REFERENCES}

1 Sigamani A, Kamath D, Xavier D. New evidence for gender disparities in cardiac interventions: CREATE-ing some clarity. Interventional 2013.

2 Shanthi M, Pekka P, Bo N. Global atlas on cardiovascular disease prevention and control. World Health Organization, 2011.

3 Bowry ADK, Lewey J, Dugani SB, et al. The burden of cardiovascular disease in low- and middle-income countries: epidemiology and management. Can J Cardiol 2015;31:1151-9.

4 Prabhakaran D, Singh K, Roth GA, et al. Cardiovascular diseases in India compared with the United States. J Am Coll Cardiol 2018;72:79-95.

5 Appelman Y, van Rijn BB, Ten Haaf ME, et al. Sex differences in cardiovascular risk factors and disease prevention. Atherosclerosis 2015;241:211-8.

6 Walli-Attaei M, Joseph P, Rosengren A, et al. Variations between women and men in risk factors, treatments, cardiovascular disease incidence, and death in 27 high-income, middle-income, and low-income countries (pure): a prospective cohort study. Lancet 2020;396:97-109.

7 Gan SC, Beaver SK, Houck PM, et al. Treatment of acute myocardial infarction and 30-day mortality among women and men. $N$ Engl J Med 2000;343:8-15.

8 Blomkalns AL, Chen AY, Hochman JS, et al. Gender disparities in the diagnosis and treatment of non-ST-segment elevation acute coronary syndromes: large-scale observations from the crusade (can rapid risk stratification of unstable angina patients suppress adverse outcomes with early implementation of the American College of Cardiology/American Heart Association guidelines) national quality improvement initiative. J Am Coll Cardiol 2005;45:832-7.

9 Shaw LJ, Shaw RE, Merz CNB, et al. American College of Cardiology-National cardiovascular data registry Investigators. impact of ethnicity and gender differences on angiographic coronary artery disease prevalence and in-hospital mortality in the American College of Cardiology-National Cardiovascular Data Registry. Circulation 2008;117:1787-801.

10 Puska P, Norrving B. Organization WH. Global atlas on cardiovascular disease prevention and control 2011.

11 Gupta R. Prevention \& control of CVD in women \& children in India. Indian J Med Res 2013;138:281-4.

12 Mahajan K, Negi PC, Merwaha R, et al. Gender differences in the management of acute coronary syndrome patients: one year results from HPIAR (HP-India ACS registry). Int J Cardiol 2017;248:1-6.

13 Pagidipati NJ, Huffman MD, Jeemon P, et al. Association between gender, process of care measures, and outcomes in ACS in India: results from the detection and management of coronary heart disease (DEMAT) registry. PLoS One 2013;8:e62061.

14 Patel A, Vishwanathan S, Nair T, et al. Sex differences in the presentation, diagnosis, and management of acute coronary syndromes: findings from the Kerala-India ACS registry. Glob Heart 2015;10:273-80.

15 India State-Level disease burden initiative CVD Collaborators. The changing patterns of cardiovascular diseases and their risk factors in the states of India: the global burden of disease study 1990-2016. Lancet Glob Health 2018;6:e1339-51. 
16 Huffman MD, Mohanan PP, Devarajan R, et al. Acute coronary syndrome quality improvement in Kerala (ACS Quik): rationale and design for a cluster-randomized stepped-wedge trial. Am Heart $J$ 2017;185:154-60.

17 Huffman MD, Mohanan PP, Devarajan R, et al. Acute coronary syndrome quality improvement in Kerala (ACS Quik) Investigators. Effect of a quality improvement intervention on clinical outcomes in patients in India with acute myocardial infarction: the ACS Quik randomized clinical trial. JAMA 2018;319:567-78.

18 Thygesen K, Alpert JS, Jaffe AS, et al. Writing group on the joint ESC/ACCF/AHA/WHF Task force for the universal definition of myocardial infarction, Thygesen K, Alpert JS, white HD, Jaffe as, Katus HA, apple FS, Lindahl B, Morrow dA, Chaitman Ba, Clemmensen PM, Johanson P, hod H, Underwood R, Bax JJ, Bonow Ro, pinto F, gibbons RJ, Fox ka, Atar D, Newby LK, Galvani M, Hamm cw, Uretsky bf, et al. third universal definition of myocardial infarction. Eur Heart J 2012;33:2551-67.

19 GUSTO investigators. An international randomized trial comparing four thrombolytic strategies for acute myocardial infarction. $N$ Engl J Med 1993;329:673-82.

20 Poon S, Goodman SG, Yan RT, et al. Bridging the gender gap: insights from a contemporary analysis of sex-related differences in the treatment and outcomes of patients with acute coronary syndromes. Am Heart J 2012;163:66-73.

21 Liakos M, Parikh PB. Gender disparities in presentation, management, and outcomes of acute myocardial infarction. Curr Cardiol Rep 2018;20:64.

22 Dey S, Flather MD, Devlin G, et al. Global registry of acute coronary events Investigators. sex-related differences in the presentation, treatment and outcomes among patients with acute coronary syndromes: the global registry of acute coronary events. Heart 2009;95:20-6.

23 Bucholz EM, Butala NM, Rathore SS, et al. Sex differences in long-term mortality after myocardial infarction: a systematic review. Circulation 2014;130:757-67.

24 Mendenhall E, Weaver LJ. Reorienting women's health in lowand middle-income countries: the case of depression and type 2 diabetes. Glob Health Action 2014;7:22803.
25 Butala NM, Desai MM, Linnander EL, et al. Gender differences in presentation, management, and in-hospital outcomes for patients with AMI in a lower-middle income country: evidence from Egypt. PLoS One 2011;6:e25904.

26 Shehab A, Bhagavathula AS, Alhabib KF, et al. Age-Related Sex Differences in Clinical Presentation, Management, and Outcomes in ST-Segment-Elevation Myocardial Infarction: Pooled Analysis of 15532 Patients From 7 Arabian Gulf Registries. J Am Heart Assoc 2020;9:e013880.

27 Song X, Chen Y, Pan W, et al. Gender based differences in patients with acute coronary syndrome: findings from Chinese registry of acute coronary events (CRACE). Chin Med J 2007.

28 Srichaiveth B, Ruengsakulrach P, Visudharom K, et al. Impact of gender on treatment and clinical outcomes in acute ST elevation myocardial infarction patients in Thailand. J Med Assoc Thai 2007;90:65-73.

29 Lu HT, Nordin R, Wan Ahmad WA, et al. Sex differences in acute coronary syndrome in a multiethnic Asian population: results of the Malaysian national cardiovascular disease databaseacute coronary syndrome (NCVD-ACS) registry. Glob Heart 2014;9:381-90.

30 Xavier D, Pais P, Devereaux PJ, et al. Treatment and outcomes of acute coronary syndromes in India (create): a prospective analysis of registry data. Lancet 2008;371:1435-42.

31 Daugherty SL, Blair IV, Havranek EP, et al. Implicit gender bias and the use of cardiovascular tests among cardiologists. J Am Heart Assoc 2017;6:6.

32 Maserejian NN, Link CL, Lutfey KL, et al. Disparities in physicians' interpretations of heart disease symptoms by patient gender: results of a video vignette factorial experiment. $J$ Womens Health 2009;18:1661-7.

33 Okunrintemi V, Valero-Elizondo J, Patrick B, et al. Gender differences in patient-reported outcomes among adults with atherosclerotic cardiovascular disease. J Am Heart Assoc 2018;7:e010498.

34 Cho L, Davis M, Elgendy I, et al. Summary of updated recommendations for primary prevention of cardiovascular disease in women: JACC state-of-the-art review. J Am Coll Cardiol 2020;75:2602-18. 